\title{
BACK PAIN IN OPHTHALMOLOGISTS
}

\author{
A. CHATTERJEE ${ }^{1}$, W. G. RYAN ${ }^{2}$ and E. S. ROSEN ${ }^{1}$ \\ Manchester
}

\begin{abstract}
SUMMARY
A questionnaire regarding the working practices and incidence of back pain was sent to all 498 consultant ophthalmologists in the United Kingdom. Three hundred and twenty-five $(65.3 \%)$ questionnaires were analysed by time spent in speciality, and time spent operating each week. One hundred and seventy-four ophthalmologists $(54 \%)$ had significant attacks of back pain, with the longest-serving consultants having an increased incidence. The number and duration of acute attacks increased with years in speciality but was unrelated to time spent operating. Treatment included analgesics in $97(56 \%)$, physiotherapy in $40(23 \%)$, and 'alternative' medical treatment including osteopathy and chiropraxy in $14(8 \%)$; no treatment was needed in $56(32 \%)$. Investigation and treatment increased with years spent in speciality, 49 (28\%) seeking medical advice, usually from a specialist, and $69(39 \%)$ requiring further investigations including plain radiographs, CT scans, myelograms and MRI scans. Nine surgeons needed surgery for back pain.
\end{abstract}

Approximately $90 \%$ of people experience back pain at least once in their life, and in the general population there is a $40-55 \%$ incidence in 1 year. ${ }^{1,2}$ This has resulted in an increasing number of days lost by employees due to sickness - over 90 million in $1990 .{ }^{3}$ Many occupations carry an increased risk of developing back pain, due to the amount of physical activity required on a regular basis. ${ }^{4}$ These include manual labour, engineering and production work, and, in the health sector, nursing. ${ }^{6}$

The aim of this study was to assess the incidence of back pain in ophthalmogists, a group of medical practitioners who, because of spending prolonged periods of time in a poor posture, may be at increased risk of developing back pain. We aimed to correlate the incidence of back pain with the working practices of ophthalmologists.

\section{METHOD}

A questionnaire regarding working practices and the inci-

From: 'Royal Eye Hospital, Manchester; '2University of Manchester. Manchester, UK.

Correspondence to: Mr A. Chatterjee, FRCSE, Manchester Royal Eye Hospital, Oxford Road, Manchester M13 9WH, UK. dence of back pain was sent to all consultant ophthalmologists in the United Kingdom. There are a total of 498 consultants and we received $336(67.5 \%)$ replies. Eleven (3\%) questionnaires were not fully completed, or the surgeons had retired. The remaining 325 questionnaires were processed.

We analysed the results with respect to the time each consultant had spent in speciality, and the time spent operating each week.

\section{RESULTS}

Of the 325 consultants who replied, 174 (54\%) had significant attacks of back pain. The longest-serving consultants, i.e. those who had spent more than 15 years in speciality, had an increased incidence of pain (Fig. 1), but this was not statistically significant (paired Student's $t$-test, $p=0.5)$. Of the 174 surgeons who experienced pain, 59 $(34 \%)$ had neck pain, $141(81 \%)$ had low back pain, and $25(14.5 \%)$ had leg pain as a worst or on!y attack. This was unrelated to time spent operating or years in ophthalmology.

Mean operating time was 8 hours per week for consultants in all groups and groups analysed by time spent operating were equally likely to have attacks of pain. Few surgeons had had more than five attacks of pain in the previous year; the number of acute attacks as well as the duration of these acute attacks increased with years in speciality but was unrelated to time spent operating. Treatment included analgesics, with 97 (56\%) taking mainly non-steroidal anti-inflammatory drugs and paracetamol. Forty $(23 \%)$ ophthalmologists required physiotherapy and $14(8 \%)$ had sought 'alternative' medical treatment including osteopathy and chiropraxy. Fifty-six (32\%) needed no treatment.

There was an increasing incidence of ophthalmologists who required investigations and treatment with increasing years spent in speciality (Fig. 2). Forty-nine (28\%) had sought medical advice, usually from a specialist, and 69 $(39 \%)$ required further investigations including plain radiographs, CT scans, myelograms and MRI scans. Nine surgeons needed surgery for back pain. The above factors were analysed for statistical significance in relation to

Eye (1994) 8, 473-474 C 1994 Royal College of Ophthalmologists 


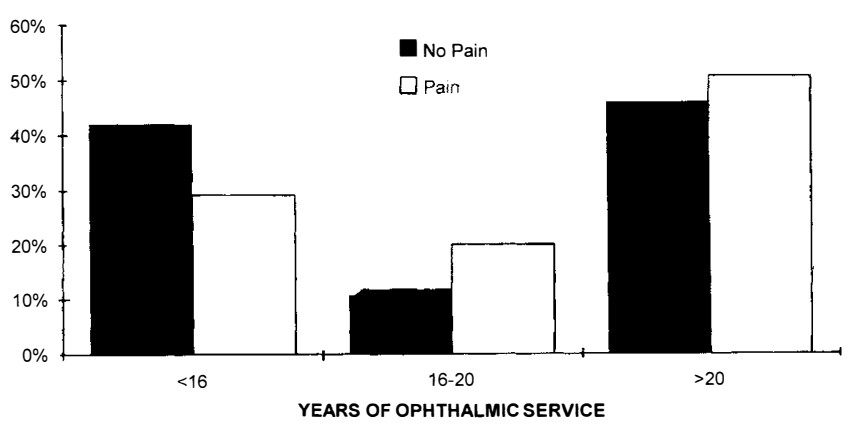

Fig. 1. Number of ophthalmologists affected by back pain in relation to years of ophthalmic service.

time in speciality and time spent operating, but neither was found to be significant.

\section{DISCUSSION}

Two categories of factors which modify the risk of back injury have been differentiated from observation: jobrelated factors such as the physical effort required, and personal factors such as the physical capability of the worker. ${ }^{6}$ Of particular risk are the following job demands:

1. General: heavy physical work.

2. Static workload: sustained trunk posture (e.g. stooping); prolonged sitting.

3. Dynamic workload: frequent bending and twisting of the trunk; lifting, lowering, pushing and pulling activities.

4. Environmental: exposure to vibration.

Of these, an ophthalmologist is particularly likely to be spending prolonged time sitting or stooping over patients whilst in theatre, and is therefore at an increased risk of developing back pain.

The incidence of pain in ophthalmologists is slightly higher than that found in the general population but the difference is not statistically significant. This may be due to the increased awareness of ophthalmologists of the problems faced in using special equipment in their daily schedule. Several respondents in the survey described

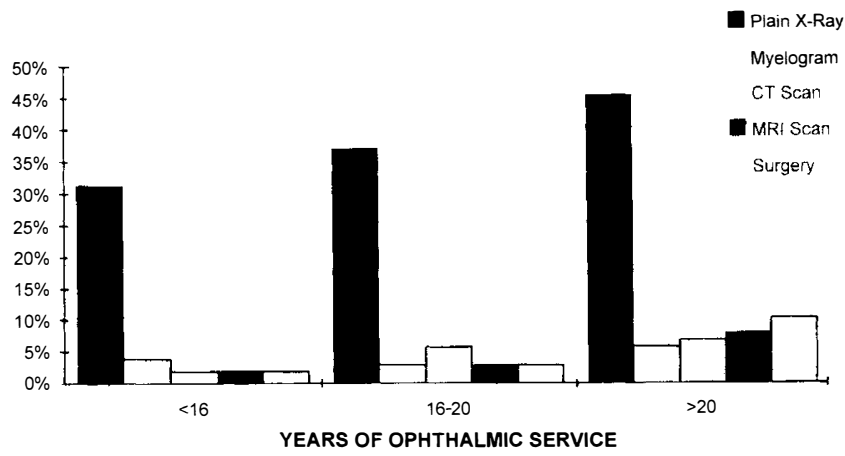

Fig. 2. Investigative treatment required for back pain in ophthalmologists in relation to years of ophthalmic service.

various methods of avoiding back pain, most of which were suggestions about maintaining a safe posture for work such as adjusting the height of the operating microscope, using well-designed chairs with backrests, and care in positioning patients on the operating table.

In conclusion, no significant difference was found between the incidence of back pain in consultant ophthalmologists as compared with the general population. Back pain is, however, a cause of considerable disability and interest.

Key words: Back pain, Doctors, Occupational disease, Ophthalmologists.

\section{REFERENCES}

1. Carey TS. Occupational back pain: issues in prevention and treatment. Baillière's Clin Rheumatol 1989;3:143-56.

2. Frank A. Low back pain. BMJ 1993;306:901-9.

3. Waddell G. Psychosocial analysis of low back pain. Baillière's Clin Rheumatol 1992;6:523-57.

4. Waddell G. A new clinical model for the treatment of low back pain. Spine 1987;12:632-44.

5. Garg M, Moore JS. Epidemiology of low back pain in industry. Occupational Med 1992;7:593-608.

6. Halpern M. Prevention of low back pain: basic ergonomics in the workplace and the clinic. Ballière's Clin Rheumatol 1992;6:705-30. 